\title{
Significance of human epidermal growth factor receptor 2 expression in colorectal cancer
}

\author{
JINHUA TU ${ }^{1,2}$, YINGHAO YU ${ }^{2}$, WEI LIU ${ }^{2}$ and SHUNPING CHEN ${ }^{2}$ \\ ${ }^{1}$ Department of Pathology, The First Affiliated Hospital of Xiamen University, Xiamen, Fujian 361004; \\ ${ }^{2}$ Department of Pathology, Dongfang Hospital, Fujian Medical University, Fuzhou, Fujian 350025, P.R. China
}

Received February 9, 2014; Accepted June 20, 2014

DOI: $10.3892 /$ etm.2014.2063

\begin{abstract}
The aim of the present study was to evaluate the protein expression level of human epidermal growth factor receptor 2 (HER-2) using immunohistochemistry (IHC), and assess the association with clinicopathological parameters and the prognosis of patients with colorectal cancer (CRC). In addition, the current study observed the consistency between the levels of HER-2 protein expression determined by IHC and HER-2 gene amplification determined by fluorescence in situ hybridization (FISH) in the CRC samples. Overexpression of HER-2 and gene amplification were examined with semiquantitative standardized IHC in 878 formalin-fixed paraffin-embedded CRC samples, while 102 of these cases were analyzed with FISH. A total of 102 cases (11.6\%), out of the 878 cases, were determined by IHC to overexpress HER-2. Of these, 25 cases were strongly positive (IHC3+), while 77 cases revealed moderate staining (IHC2+). HER-2 overexpression was more frequent in early-stage cases compared with advanced-stage cases of CRC $(\mathrm{P}<0.001)$. However, there was no association observed between HER-2 overexpression and clinicopathological parameters. FISH analysis revealed that $64 \%(16 / 25)$ of the IHC3+ cases had HER-2 gene amplification. By contrast, only $6.5 \%(5 / 77)$ of the IHC $2+$ cases, and none of the 20 randomly selected IHC0 or $1+$ cases, demonstrated HER-2 gene amplification. Furthermore, no associations were observed between HER-2 overexpression or gene amplification with the survival time. Thus, the present study observed that HER-2 overexpression does not correlate with other clinicopathological data or the survival rate, with the exception of clinical stages. However, IHC3+ and 2+ cases should be further analyzed by FISH to assess the status of the HER-2 gene in
\end{abstract}

Correspondence to: Professor Yinghao Yu, Department of Pathology, Dongfang Hospital, Fujian Medical University, 156 North Road, Fuzhou, Fujian 350025, P.R. China

E-mail: jinhuatu@163.com

Key words: colorectal cancer, human epidermal growth factor receptor 2, immunohistochemistry, fluorescence in situ hybridization, herceptin
CRC. Patients with HER-2 gene amplification may constitute as potential candidates for targeted therapy with trastuzumab.

\section{Introduction}

Colorectal cancer (CRC) is one of the most common types of tumor and is the fourth leading cause of cancer mortality worldwide (1). With economic development and changes in lifestyle, the incidence and mortality rates of CRC have rapidly increased in China. With the exception of surgery, the majority of treatments for CRC, including traditional chemoand radiation therapies, are not fully efficacious in treating the disease. The recent development of novel drugs targeting CRC has improved the survival rate of patients with the disease. Targeted drugs for the treatment of cancer have rapidly developed. The human epidermal growth factor receptor 2 (HER-2) signaling pathway plays an important role in tumor proliferation, angiogenesis, differentiation and metastasis in CRC (2). HER-2-targeted drugs, including Herceptin, have been developed and widely applied for the treatment of breast cancer presenting with membranous HER-2 overexpression $(3,4)$.

The HER-2 oncogene is a member of the tyrosine kinase family of receptors, which includes HER-1, also known as epidermal growth factor receptor (EGFR), HER-2, HER-3 and HER-4. HER-2 is located on chromosome 17q21 and encodes a $185 \mathrm{kDa}$ transmembrane protein. HER-2 activation initiates signal cascades, including the mitogen-activated protein kinase and phosphoinositide 3-kinase/Akt signaling pathways, which are essential for cell proliferation and differentiation. Thus, HER-2 overexpression leads to the disordered proliferation and malignant transformation of cells. HER-2 is overexpressed in numerous types of malignant cancer, including breast, ovarian, gastric, lung, colorectal and prostate cancers (5). Chen et al (6) revealed that the detection of HER-2 protein expression may be used to assess the malignant biological behavior and prognosis of gastric cancer. The European Committee has already approved chemotherapy combined with Herceptin as a treatment for cases of gastric cancer presenting with membranous HER-2 overexpression (7). Thus, ensuring that the expression level of HER-2 in patients with gastric cancer is examined accurately is of importance. However, conflicting data exist with regard to the prevalence of HER-2 overexpression in CRC, with a range between 2 and $47 \%$, while the prevalence of HER-2 gene amplification ranges between 2.5 and 7.4\% (8-27). 
Similarly, there is controversy in the published literature with regard to the association between the survival rate and HER-2 overexpression in CRC (10-13,28).

Since there are few published studies investigating HER-2 expression in CRC and genetic differences exist between ethnic groups with regard to tumorigenesis, numerous topics require further study. Thus, the present study investigated the frequency of HER-2 overexpression and gene amplification in CRC, and whether HER-2 overexpression and gene amplification were consistent. In addition, associations between HER-2 overexpression with clinicopathological parameters and the prognosis of CRC were analyzed.

\section{Materials and methods}

Patients and tissue specimens. Clinicopathological data and paraffin-embedded specimens were collected from 878 patients who underwent colorectal resections at Dongfang Hospital (Fuzhou, China) between January 2006 and April 2012. The study was approved by the ethics committee of Dongfang Hospital and written informed patient consent was obtained from the patient or the patient's family. Of the 878 patients, 541 were male and 337 were female, with ages ranging between 17 and 85 years (median age, 51 years). A total of 396 tumors were located in the rectum, while 482 tumors were in the colon. None of the patients had received preoperative neoadjuvant chemotherapy or radiotherapy. A total of 100 paraneoplastic normal tissue specimens of CRC were used as controls.

The conditions of the patients were assessed according to the Tumor Node and Metastasis (TNM) Classification of Malignant Tumors (29). TNM classification revealed that $490(55.8 \%)$ patients were at stages 0 , I or II, while $388(44.2 \%)$ patients were at stages III or IV. One tumor was classified as pTis, 148 tumors were pT1, 341 tumors were pT2, 314 tumors were pT3 and 74 tumors were pT4. The World Health Organization Classification of Tumors was used for histological classification (30). A total of 761 (86.7\%) tumors were classified as well and moderately differentiated, while $117(13.3 \%)$ were poorly differentiated. All the specimens were routinely fixed in $10 \%$ formalin, embedded in paraffin and verified pathologically prior to inclusion in the present study. Follow-up was conducted at 6, 12, 18 and 24 months following surgery and in one-year intervals thereafter. Patients who succumbed within four weeks following radical surgery and those who were $>85$-years-old were excluded from the current analysis.

Immunohistochemical staining. HER-2 overexpression analysis was conducted on $4-\mu \mathrm{m}$-thick sections. Briefly, following deparaffinization and rehydration, the tissue samples were incubated in a citrate buffer solution at $90-95^{\circ} \mathrm{C}$ for $20 \mathrm{~min}$. The slides were washed with phosphate-buffered saline (PBS) for three times for $3 \mathrm{~min}$. Endogenous peroxidase activity was suppressed by a $10 \mathrm{~min}$ incubation in methanol with $3 \%$ hydrogen peroxide. A primary monoclonal rabbit antibody against the human HER-2 protein (Clone SP3; Lab Vision Corporation, Fremont, CA, USA) was applied for $60 \mathrm{~min}$ at room temperature. Subsequently, a secondary goat anti-rabbit antibody (Lab Vision Corporation) conjugated to horseradish peroxidase was applied for $30 \mathrm{~min}$ at room temperature. The bound antibody was visualized using a peroxidase chromogen substrate. The sections were counterstained with hematoxylin and a coverslip was applied. Paraffin slides of invasive breast carcinomas were used as a positive control (these were obtained from the Department of Pathology, Dongfang Hospital). For antibody-negative controls, the primary antibodies were substituted with PBS.

Slides were examined separately by two independent pathologists blinded to each others results. Discrepancies between the investigators ( $<5 \%$ of cases) required a third joint observation with a conclusive agreement. The HercepTest ${ }^{\mathrm{Tw}}$ scoring system specific to gastric cancer was used to determine tumor cell reactivity, as described by Hofmann et al (31) in 2008. No reactivity or membranous reactivity in $<10 \%$ of the tumor cells was defined as an immunohistochemistry (IHC) score of 0; faint/barely perceptible partial membrane reactivity in $>10 \%$ of the tumor cells was defined as a score of $1+$; weak to moderate complete or basolateral membranous reactivity in $>10 \%$ of the tumor cells was defined as a score of 2+; strong complete or basolateral membranous reactivity in $>10 \%$ of the tumor cells was defined as a score of $3+$. A score of 0 or $1+$ was considered negative, while a score of $2+$ or $3+$ was considered positive. Cytoplasmic staining may have been present, but was not included in the determination of positivity.

Fluorescence in situ hybridization (FISH). FISH analysis was applied to all $\mathrm{IHC} 2+$ and $3+$ tumors, as well as to 20 randomly selected IHCO and $1+$ cases. Paraffin slides of invasive breast cancers were used as a positive control. FISH was conducted with a HER-2 DNA Probe kit (GP Medical Technologies, Ltd., Beijing, China), according to the manufacturer's instructions. The commercially available double-color FISH probe consisted of two probes: 17q11.2-q12 (labeled with a red signal) covering the whole HER-2 gene and the control, centromeric chromosome 17p11.1-q11.1 (labeled with a green signal).

The FISH-fixed glass slides with $4-\mu \mathrm{m}$-thick sections were heated overnight at $65^{\circ} \mathrm{C}$, deparaffinized in two 10 -min changes of xylene, rehydrated with two 3 -min changes of $100 \%$ ethanol, one $3 \mathrm{~min}$ change of $85 \%$ ethanol and one 3 min change of $70 \%$ ethanol, and immersed for $15 \mathrm{~min}$ in pure water at $90^{\circ} \mathrm{C}$. The slides were incubated (in a water bath) for $35 \mathrm{~min}$ in sodium sulfite acid at $50^{\circ} \mathrm{C}$ and washed in $2 \mathrm{X}$ sodium saline citrate ( $\mathrm{SSC} ; \mathrm{pH} 7.2)$ at room temperature. The slides were incubated for $12 \mathrm{~min}$ in proteinase $\mathrm{K}$ solution at $37^{\circ} \mathrm{C}$, washed in $2 \mathrm{X} \mathrm{SSC}(\mathrm{pH} \mathrm{7.2)}$ at room temperature, dehydrated with 70,85 and $100 \%$ ethanol and allowed to air-dry. To denature the DNA, the slides were placed in $78.5^{\circ} \mathrm{C}$ preheated $70 \%$ formamide/2X SSC for $8 \mathrm{~min}$ and dehydrated in a graded series of ethanol concentrations that had been precooled to $-20^{\circ} \mathrm{C}$. Following air-drying, $10 \mu \mathrm{l}$ probe, which had been previously destructured at $75.5^{\circ} \mathrm{C}$ for $7 \mathrm{~min}$, was applied onto each slide. A cover slip was placed and sealed with rubber cement, and the slides were hybridized at $42.8^{\circ} \mathrm{C}$ overnight. After $16-18 \mathrm{~h}$ hybridization, the slides were washed in $46^{\circ} \mathrm{C}$ preheated post-hybridization buffer (2X SSC $/ 0.1 \%$ sodium dodecyl sulfate) for $5 \mathrm{~min}$ and rinsed in $70 \%$ ethanol. Following air-drying (out of 
A

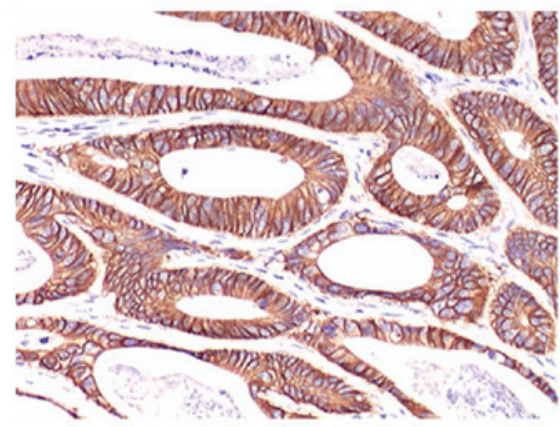

C

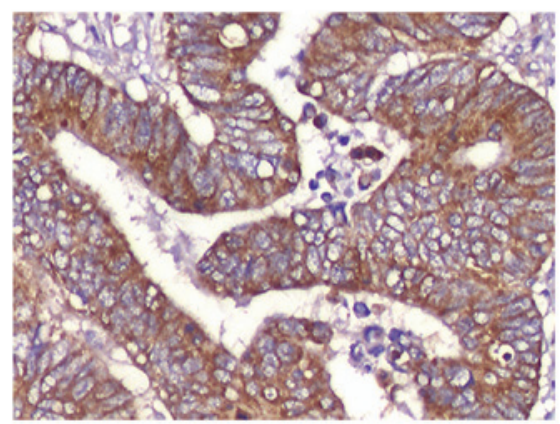

B

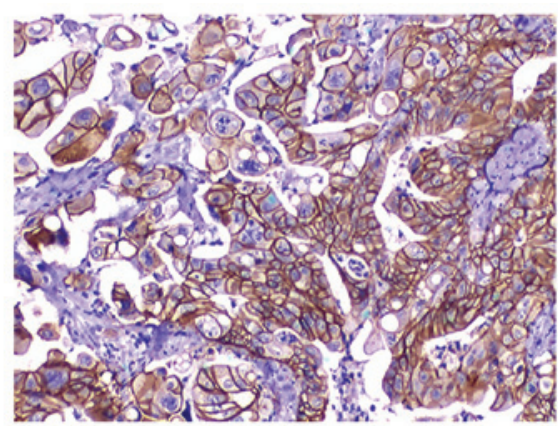

D

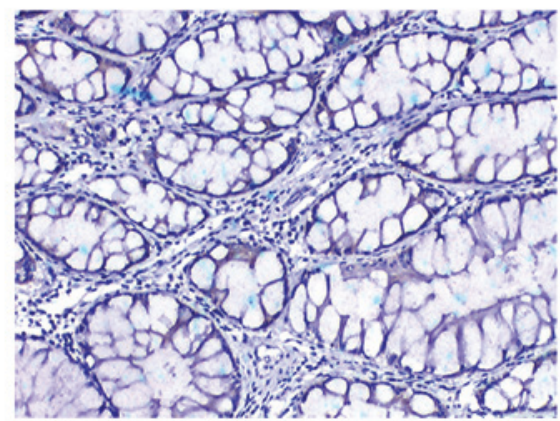

Figure 1. Immunohistochemical staining for human epidermal growth factor receptor 2 (HER-2). (A) HER-2 (IHC3+), well differentiated colorectal cancer (CRC; magnification, x200). (B) HER-2 (IHC3+), moderately and poorly differentiated CRC (magnification, x200). (C) HER-2 (IHC2+), well and moderately differentiated CRC (magnification, x200). (D) HER-2 was negative in normal epithelial cells adjacent to the tumor tissue (magnification, x100).
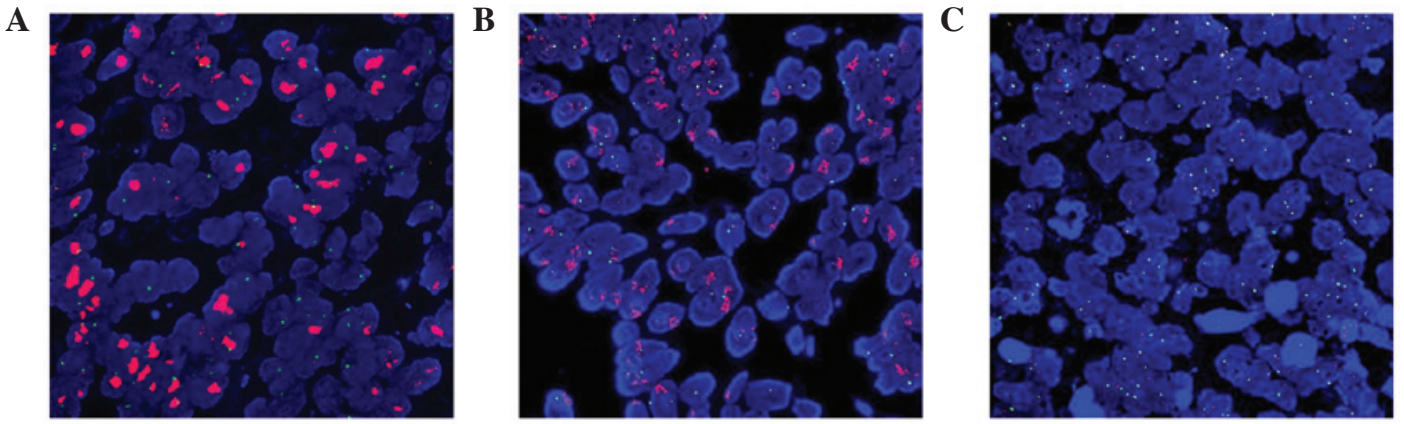

Figure 2. Fluorescence in situ hybridization analysis of human epidermal growth factor receptor 2 (HER-2) gene amplification (magnification, $\mathrm{x} 1,000)$. (A) Positive amplification of the HER-2 gene, as indicated by red cluster signals in the tumor cells. (B) Positive amplification of the HER-2 gene; the ratio of the HER-2 gene (red signals) to centromeric probe 17 (green signals) was >2.2. (C) Negative amplification of the HER-2 gene; the ratio of the HER-2 gene (red signals) to CEP 17 (green signals) was $<1.8$.

direct light), the slides were counterstained with $10 \mu \mathrm{l}$ 4',6-diamidino-2-phenylindole/anti-fade solution and coverslips were applied.

After $10 \mathrm{~min}$, the slides were observed under a fluorescence microscope (Olympus BX51; Olympus, Tokyo, Japan). A total of 30 randomly selected tumor nuclei were evaluated in three separate, distinct microscopic areas. Cases were classified as negative/no amplification when the HER-2 gene (red signal) to centromeric probe 17 (green signal) ratio was $<1.8$, while cases with a ratio of $>2.2$ were classified as positive/amplification. When the ratio was between 1.8 and $2.2, \geq 100$ randomly selected tumor nuclei were evaluated. Furthermore, a cell was considered to demonstrate amplification when a definite cluster or $>10$ red signals for the HER-2 gene were identified, as described in a previous FISH study (32). Cases were defined as chromosome 17 polysomy when the green signal was $>2.25$ in each nucleus when counting $\geq 30$ tumor nuclei.
Statistical analysis. The $\chi^{2}$ test was performed to analyze the association between HER-2 overexpression and the clinicopathological characteristics of CRC, and the correlation between IHC and FISH. The probability of survival for the various subgroups was calculated using the Kaplan-Meier method. All statistical analyses were performed two-sided, where $\mathrm{P}<0.05$ was considered to indicate a statistically significant difference. SPSS 16.0 software (SPSS, Inc., Chicago, IL, USA) was used for analysis.

\section{Results}

Overexpression of HER-2. A total of 102 cases (11.6\%) out of the 878 patients were demonstrated to have overexpressed HER-2 by IHC. Of these, 25 cases were strongly positive (3+; Figs. 1A and B) and 77 cases revealed moderate staining (2+; Fig. 1C). HER-2 overexpression was more frequent in 0 , I and II stage cases compared with stage III and IV cases 
Table I. Association between HER-2 overexpression (IHC3+ and 2+) and the clinicopathological characteristics of CRC.

\begin{tabular}{|c|c|c|c|}
\hline Clinicopathological characteristic & Cases, $\mathrm{n}$ & HER-2 overexpression, n (\%) & P-value \\
\hline \multicolumn{4}{|l|}{ Gender } \\
\hline Male & 541 & $64(11.8)$ & \multirow[t]{2}{*}{0.803} \\
\hline Female & 337 & $38(11.3)$ & \\
\hline \multicolumn{4}{|l|}{ Age, years } \\
\hline$<60$ & 443 & $51(11.5)$ & \multirow[t]{2}{*}{0.922} \\
\hline$\geq 60$ & 435 & $51(11.7)$ & \\
\hline \multicolumn{4}{|l|}{ Tumor site } \\
\hline Colon & 482 & $50(10.4)$ & \multirow[t]{2}{*}{0.204} \\
\hline Rectum & 396 & $52(13.1)$ & \\
\hline \multicolumn{4}{|l|}{ Tumor size, $\mathrm{cm}$} \\
\hline$<5$ & 445 & $49(11.0)$ & \multirow[t]{2}{*}{0.570} \\
\hline$\geq 5$ & 433 & $53(12.2)$ & \\
\hline \multicolumn{4}{|l|}{ Depth of invasion } \\
\hline Tis+T1 & 12 & $0(0.0)$ & \multirow[t]{4}{*}{0.514} \\
\hline $\mathrm{T} 2$ & 174 & $20(11.5)$ & \\
\hline $\mathrm{T} 3$ & 648 & $79(12.2)$ & \\
\hline $\mathrm{T} 4$ & 44 & $3(6.8)$ & \\
\hline \multicolumn{4}{|l|}{ Classification } \\
\hline Well and moderate & 761 & $94(12.4)$ & \multirow[t]{2}{*}{0.083} \\
\hline Moderate and poor & 117 & $8(6.8)$ & \\
\hline \multicolumn{4}{|l|}{ TNM stage } \\
\hline 0/I/II & 490 & $79(16.1)$ & \multirow[t]{2}{*}{0.000} \\
\hline III/IV & 388 & $23(5.9)$ & \\
\hline \multicolumn{4}{|l|}{ Lymph node metastases } \\
\hline N0 & 513 & $56(10.9)$ & \multirow[t]{3}{*}{0.611} \\
\hline N1 & 229 & $27(11.8)$ & \\
\hline $\mathrm{N} 2$ & 136 & $19(14.0)$ & \\
\hline \multicolumn{4}{|l|}{ Distant metastases } \\
\hline M0 & 804 & $92(11.4)$ & \multirow[t]{2}{*}{0.595} \\
\hline M1 & 74 & $10(13.5)$ & \\
\hline
\end{tabular}

TNM, tumor node and metastsis; HER-2, human epidermal growth factor receptor 2; IHC, immunohistochemsistry; CRC, colorectal cancer.

$(\mathrm{P}<0.001)$. No association was observed between HER-2 overexpression and gender, age, tumor site, size, depth of invasion, lymph node metastases or distant metastases $(\mathrm{P}>0.05$; Table I). Stromal and normal epithelial cells adjacent to the tumor tissue were negative (Fig. 1D).

HER-2 gene amplification. Following FISH analysis, 24.5\% $(25 / 102)$ of the IHC3+ cases were shown to exhibit HER-2 gene amplification (Fig. 2A). By contrast, only 6.5\% (5/77) of IHC2+ cases (Fig. 2B), and none of the randomly selected 20 cases with IHC0/1+, demonstrated HER-2 gene amplification (Fig. 2C). A relatively high level of consistency was observed between IHC3+ and IHC0/1+ with FISH (64 and 100\%, respectively); however, there was a low level of consistency with the results between IHC2+ and FISH (6.5\%; Table II).

Chromosome 17 polysomy and non-polysomy. Chromosome 17 copy number analysis was applied to all $\mathrm{IHC} 2+$ and $3+$ cases.
Two cases $(8 \%)$ revealed chromosome 17 polysomy out of 25 IHC3 + cases, while only one case $(1 \%)$ was identified in the 77 IHC2+ cases. Among the 21 tumors with HER-2 gene amplification, only one case (5\%) exhibited chromosome 17 polysomy, while two cases $(2.5 \%)$ were observed in the 81 cases without HER-2 gene amplification. In the FISH-positive cases, there was one case $(6.3 \%)$ of chromosome 17 polysomy in 16 IHC3+ cases and no chromosome 17 polysomy observed in the five $\mathrm{IHC} 2+$ cases. With regard to the FISH-negative cases, one case $(11 \%)$ out of nine IHC3+ cases and one case (1\%) out of the 72 IHC2+ cases had chromosome 17 polysomy (Table III).

Survival analysis. Follow-up was conducted on 349 cases, including 46 HER-2-positive (IHC3+ and 2+) and 303 HER-2-negative (IHCO and 1+) cases. Among the 349 cases, 202 were early-stage ( 0 , I or II stage; HER-2-positive, 25; HER-2-negative, 177) and 147 were advanced stage cases 
Table II. Concordance analysis between HER-2 overexpression and amplification.

\begin{tabular}{lcccr}
\hline & & \multicolumn{2}{c}{ FISH, $\mathrm{n}(\%)$} & \\
\cline { 3 - 4 } IHC status & Cases, $\mathrm{n}$ & Amplification & No amplification & Concordance $(\%)$ \\
\hline IHC3+ & 25 & $16(64.0)$ & $9(36.0)$ & 64.0 \\
IHC2+ & 77 & $5(6.5)$ & $72(93.5)$ & 6.5 \\
IHC0/1+ & 20 & $0(0.0)$ & $20(100.0)$ & 0.0 \\
Positive control & 10 & $10(100.0)$ & $10(0.0)$ & 100.0 \\
\hline
\end{tabular}

IHC, immunohistochemistry; FISH, fluorescence in situ hybridization; HER-2, human epidermal growth factor receptor 2.

A
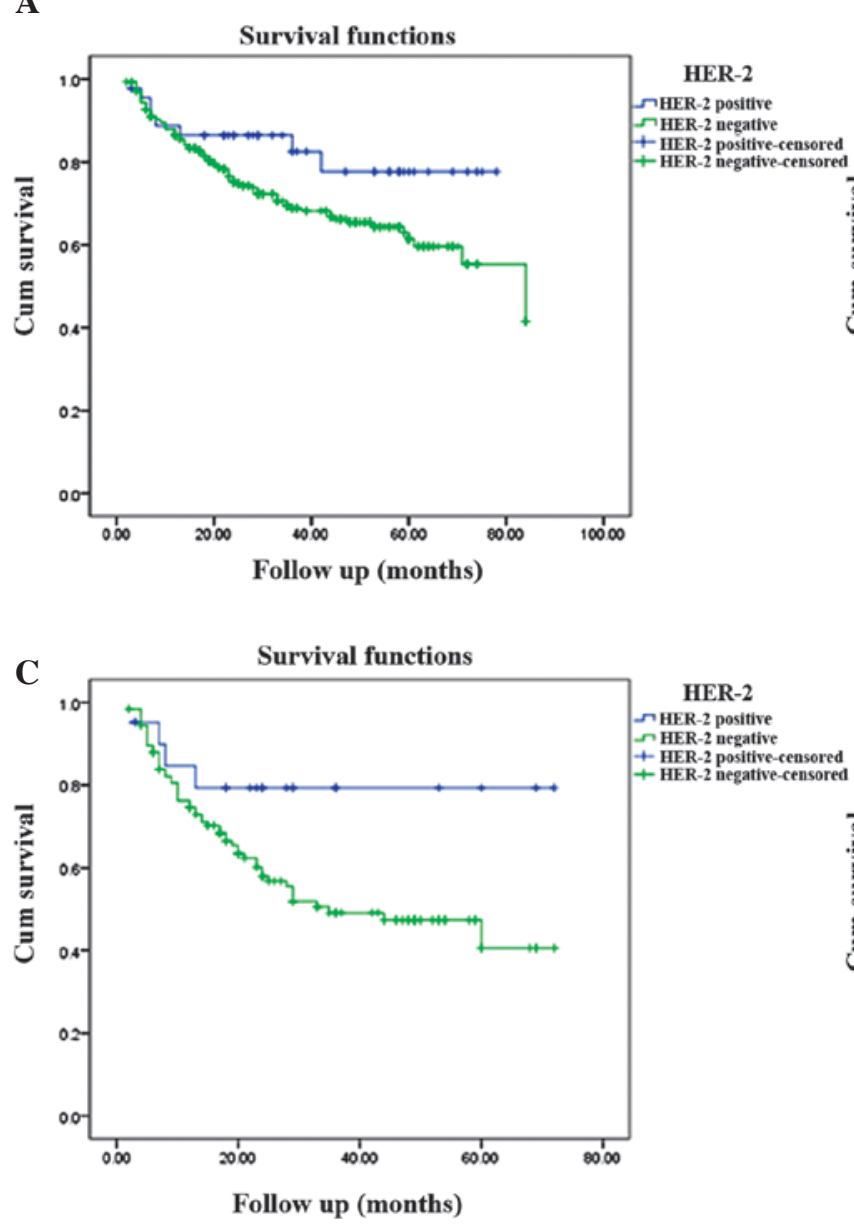

B

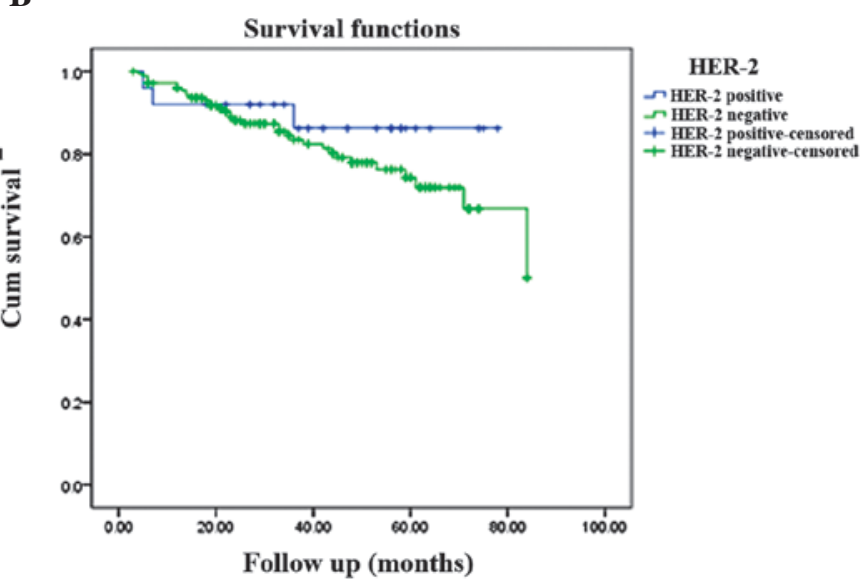

D

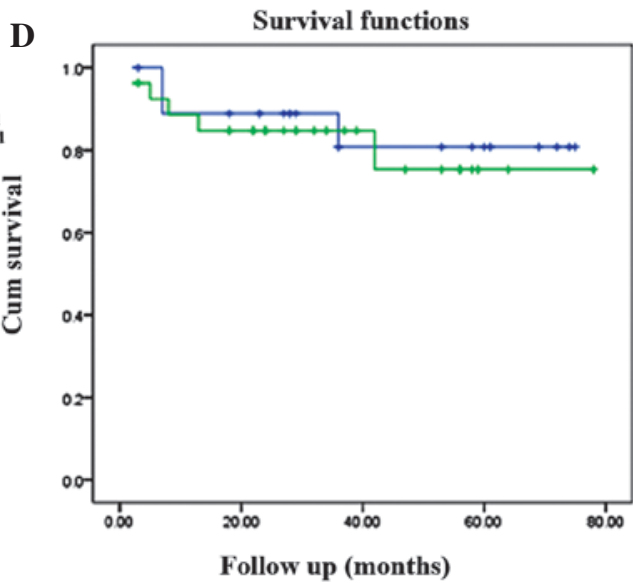

HER-2

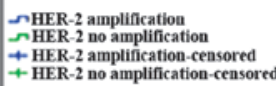

Figure 3. Kaplan-Meier survival analysis comparing HER-2 positivity [immunohistochemistry (IHC)3+ and 2+] with HER-2 negativity in (A) colorectal cancer (CRC; $\mathrm{P}=0.082)$, (B) early-stage CRC ( $\mathrm{P}=0.328)$ and (C) advanced-stage $\mathrm{CRC}(\mathrm{P}=0.06)$. (D) Kaplan-Meier survival analysis comparing HER-2 amplification with HER-2 non-amplification in HER-2-positive cases ( $\mathrm{P}=0.736$ ). Cum, cumulative; HER-2, human epidermal growth factor receptor 2.

(III or IV stage; HER-2-positive, 21; HER-2-negative, 126). The median follow-up duration was 28 months (range, 2-84 months) and 135 cases were followed for $>3$ years. The median survival time was 84 months and the mean survival time was 60.9 months. The mean survival times of the HER-2-positive and -negative groups were 64.9 and 59.5 months, respectively. The HER-2-positive CRC patients exhibited higher three- and five-year survival rates compared with HER-2-negative patients (77.7 vs. $68.8 \%$ and 77.7 vs. $61.4 \%$, respectively); however, the difference was not statistically significant ( $\mathrm{P}=0.082$; Fig. 3).
HER-2-positive patients with early and advanced stage CRC revealed higher survival rates compared with HER-2-negative cases at three years ( 86.2 vs. $83.5 \%$ and 79.4 vs. $49.1 \%$, respectively) and five years (86.2 vs. $74.3 \%$ and 79.4 vs. $40.6 \%$, respectively); however, this difference was also not statistically significant $(\mathrm{P}=0.328$ and $\mathrm{P}=0.06$, respectively; Fig 3 ). A total of 20 HER-2 gene amplification and 26 gene non-amplification cases were included in the 46 HER-2-positive cases. In the HER-2-positive group, HER-2 gene amplification and non-amplification exhibited a three-year survival rate of 80.8 
Table III. Association between chromosome 17 copy number and HER-2 overexpression/amplification.

Chromosome 17 copy number,

\begin{tabular}{lccc}
\multirow{2}{*}{$\begin{array}{l}\text { IHC/FISH } \\
\text { status }\end{array}$} & Cases, $\mathrm{n}$ & Polysomy & Non-polysomy \\
\cline { 3 - 4 } IHC3+ & 25 & $2(8)$ & $23(92)$ \\
IHC2+ & 77 & $1(1)$ & $76(99)$ \\
FISH+ & 21 & $1(4.8)$ & $20(95.2)$ \\
FISH- & 81 & $2(2.5)$ & $79(97.5)$ \\
IHC3+/FISH+ & 16 & $1(6.3)$ & $15(93.7)$ \\
IHC2+/FISH+ & 5 & $0(0)$ & $0(100)$ \\
IHC3+/FISH- & 9 & $1(11)$ & $8(89)$ \\
IHC2+ /FISH- & 72 & $1(1)$ & $71(99)$
\end{tabular}

IHC, immunohistochemistry; FISH, fluorescence in situ hybridization; HER-2, human epidermal growth factor receptor 2.

vs. $84.7 \%$, respectively, and five-year survival rate of 80.8 vs. $75.3 \%$, respectively (Fig 3); however, this difference was not statistically significant $(\mathrm{P}=0.736)$. In general, there was no association between HER-2 overexpression or gene amplification and survival time.

\section{Discussion}

In the present study, HER-2 overexpression was observed in 102 (11.6\%) of the 878 Chinese CRC samples. Previous studies have reported positive rates of HER-2 overexpression in CRC ranging between 2 and 47.4\%. The positive rates of HER-2 overexpression may have varied in these studies due to differences in the IHC procedure, sample size and the scoring system employed. Park et al (10) revealed HER-2 overexpression in $47.4 \%$ of 137 patients with CRC, whereas Antonacopoulou et al (16) observed overexpression in $24.7 \%$ of 124 patients using IHC performed on whole sections. Demirbas et al (11) demonstrated HER-2 overexpression in 9.6\% of 104 patients with CRC using tissue microarray (TMA). The results of these studies indicate that the expression of HER-2 in CRC is associated with the prognosis and may constitute a potential candidate for novel adjuvant therapies involving humanized monoclonal antibodies, such as Herceptin. However, other studies have demonstrated that the expression of HER-2 in CRC was not associated with the prognosis, based on a subjunctive scoring system of IHC. Kruszewski et al (20) reported HER-2 overexpression in 27\% of 202 CRC patients, while Kavanagh et al (12) observed overexpression in $11 \%$ of 132 patients using IHC performed on whole sections. Kim et al (23) reported HER-2 overexpression in $0.5 \%$ of 185 patients with CRC, and Marx et al (24) reported overexpression in $2.7 \%$ of 1,851 patients using TMA. Furthermore, a number of studies have demonstrated that HER-2 overexpression was not associated with gender, age, histological tumor type, tumor localization, grading, $\mathrm{pT}, \mathrm{pN}$, pM or survival $(12,22,28)$.

Consequently, there are two hypotheses on the role of HER-2 expression in CRC at present. Firstly, HER-2 overex- pression may be an independent prognostic factor in CRC, whilst secondly, expression of HER-2 in CRC is not associated with prognosis. No associations between HER-2 overexpression and gender, age, tumor site, size, depth of invasion, lymph node metastases or distant metastases $(\mathrm{P}>0.05)$ were observed in the present study. Furthermore, no statistically significant difference was observed between HER-2 amplification and HER-2 non-amplification $(\mathrm{P}=0.736)$ in the three- and five-year survival rates. Thus, the current data were consistent with the latter hypothesis that HER-2 overexpression is not an independent prognostic factor of CRC. However, the present study also revealed that HER-2 overexpression was associated with the TNM stage. Early-stage cancers exhibited a higher rate of HER-2 overexpression compared with advanced-stage cancers (16.1 vs. $5.9 \% ; \mathrm{P}<0.001)$. However, this observation is not consistent with those of previous studies where the HER-2 positivity rate of early-stage cancers was lower than that of advanced-stage tumors $(16,33)$, or where the HER-2 positivity rate of cancers was shown not be associated with the TNM stage $(12,20,24,26,34)$.

A previous study demonstrated that Herceptin, an anti-HER-2 monoclonal antibody, inhibits HCA-7 cell proliferation in vitro and in vivo (35). As the first HER-2 dimerization inhibitor, pertuzumab (a monoclonal antibody), also exhibits antitumor activity on human colon cancer cells in vitro and in vivo, in particular when combined with erlotinib (36). A phase II trial revealed that a low overexpression rate of HER-2 (8.0\%) in advanced CRC limits the application of Herceptin as a treatment for advanced-stage CRC; however, partial responses were observed in five of the seven evaluable patients (17). Annually, there are $\sim$ one million new cases of CRC worldwide, indicating that of these, 100,000 cases may overexpress HER-2, according to the HER-2 positivity rate of $11.6 \%$ in the present study. HER-2 gene amplification is vital for targeted tumor therapy, such as Herceptin for breast tumors. However, not all HER-2-positive cases exhibit HER-2 gene amplification.

In the current study, HER-2 gene amplification was observed in $21 \%(21 / 102)$ of the tumors exhibiting HER-2 overexpression and in $2.4 \%$ of the total 878 cases of CRC. These results were similar to those from previous studies where the HER-2 gene amplification rate ranged between 2.5 and $7.4 \%(11,19,24)$. Liu et al (37) reported that the rate of consistency between IHC and FISH was 70\% for IHC3+ and $14 \%$ for IHC2+ in gastric cancer samples. In the present study, a relatively high consistency rate was observed between IHC3+ and IHC0/1+ with FISH (64 and 100\%, respectively); however, there was a low consistency result between IHC2+ and FISH (6.5\%). Thus, the concordance rate between IHC and FISH in $\mathrm{CRC}$ is analogous to that observed in gastric cancer.

A number of studies have demonstrated that chromosome 17 polysomy may be the main reason for HER-2 overexpression but not HER-2 gene amplification (37-39). In the present study, only one case (11\%) of chromosome 17 polysomy was observed out of nine IHC3+ cases with no HER-2 gene amplification. In addition, there was only one case of chromosome 17 polysomy in the $72 \mathrm{IHC} 2+$ cases with no HER-2 gene amplification. Thus, it was hypothesized that chromosome 17 polysomy may not be the reason for HER-2 positivity without HER-2 gene amplification in CRC. It may be that different mechanisms result 
in HER-2 overexpression, including transcriptional activation by other genes, post-transcriptional events or a new genomic environment associated with amplification (40-42). However, it is considered that the inconsistency between HER-2 overexpression and gene amplification is associated with the two methods, namely of immunohistochemical staining and fluorescence in situ hybridization.

IHC is less expensive and time-consuming, easy to store and perform, and requires a routinely available microscope. However, the IHC techniques may be potentially affected by a number of variables, including tissue fixation, processing, primary antibody selection, detection systems and methods of antigen retrieval. Furthermore, as the proposed scoring system for IHC is subjective, interpretation may vary among observers. These factors, in addition to the small study sample sizes, may also account for the variable rates of HER-2 immunoreactivity, as well as the conflicting results indicating that HER-2 is associated with adverse clinical outcomes in certain studies, but not in others. At present, FISH is regarded as the most effective method for the detection of HER-2 amplification, as it is has high rates of sensitivity and specificity. FISH is also advantageous as it can be conducted with small tumor samples and with formalin-fixed and paraffin-embedded tissue samples. This technique allows for the direct visualization of gene amplification in the nuclei and provides an objective count of genes and chromosomes on a cell-by-cell basis. However, this method is expensive, time-consuming, requires a fluorescent microscope and is difficult to separate in situ and invasive carcinomas. Furthermore, fluorescence fades rapidly; thus, a permanent record is not created $(32,43,44)$.

CRC involves changes in multiple oncogenes, tumor suppressor genes and signal transduction pathways. Almost all tumors with more than one locus are involved in tumorigenesis. EGFR inhibitors have been widely used in oncotherapy. The identification of the mutant kirsten rat sarcoma viral oncogene homolog (KRAS) as a predictor of resistance to EGFR monoclonal antibodies created a major change in the treatment of CRC (43-45). As it is known drug resistance results in the failure of chemotherapy and poor prognosis. However, it also remains a cause for limiting the EGFR inhibitor long-term efficacy, with the exception of the KRAS mutant that plays a vital role in predicting EGFR monoclonal antibodies in CRC. EGFR inhibitor resistance is associated with the mechanisms that follow signal pathway activation of HER-2, VEGF and platelet-derived growth factor. In other words, all of these are activated by circumventing EGFR protein tyrosine kinase signaling pathway, activation. Herreros-Villanueva et al (25) hypothesized that HER-2 gene amplification may be one of the causes of insensitivity to anti-EGFR therapies, including cetuximab. The study reported that HER-2 gene amplification was observed in $26.3 \%$ of KRAS and v-raf murine sarcoma viral oncogene homolog B (BRAF) wild type colorectal carcinomas in Spanish patients. In addition, previous studies have reported that KRAS and BRAF mutations are mutually exclusive in CRC; if there are KRAS mutations, no BRAF mutations are present, and vice versa $(45,46)$. The KRAS mutation statuses in 280 samples selected from 878 patients with CRC were detected in a previous study (47). The results revealed that there were no cases of HER-2 gene amplification in KRAS mutant types, with all HER-2 gene amplification occurring in the KRAS wild type. Considering that there were no cases of the KRAS mutation type in the patients with CRC, whether KRAS mutations and HER-2 gene amplification are mutually exclusive remains to be elucidated. Furthermore, the association between BRAF mutations and HER-2 gene amplification requires further investigation, as well as whether HER-2 monoclonal antibodies may be used to aid EGFR inhibitor resistance in CRC.

As the standard treatment for breast and gastric cancers, the premise of the success of Herceptin is concordance between HER-2 expression and gene amplification. A previous study revealed that lapatinib, an EGFR/HER-2 kinase inhibitor, combined with Panobinostat, a histone deacetylase inhibitor, interacted synergistically to inhibit the proliferation and colony formation in all CRC cell lines tested. Compared with either agent alone, there was no apparent increase in toxicity (48). A phase II trial revealed that Herceptin exerted a therapeutic effect on CRC, although the low overexpression rate of HER-2 (8.0\%) in advanced CRC limited the efficacy of the drug (17). Chen et al (49) revealed that there was no statistically significant difference in HER-2 expression between colorectal liver metastases and the corresponding primary tumors. Thus, metastatic lesions may also be suitable for anti-HER-2 therapy due to the homogenicity of HER-2 expression in CRC. These results indicate that HER-2 may be a promising target as an adjuvant therapy for patients with CRC. However, to determine the precise curative effect of anti-HER-2 therapy on CRC, multicenter or international cooperation is required, through large clinical trials, to study the association between the HER-2 and KRAS genes.

In conclusion, HER-2 overexpression and gene amplification are present in CRC. With the exception of clinical stages, no associations were observed between HER-2 overexpression and other clinicopathological data in the present study. HER-2 overexpression and gene amplification did not correlate with established prognostic indicators. However, IHC3+ and 2+ cases should be further analyzed by FISH to assess the gene status of HER-2 in CRC. Patients with HER-2 gene amplification may be potential candidates for targeted therapy with Herceptin. However, further studies are required to confirm these results.

\section{References}

1. Ferlay J, Shin H, Bray F, et al: GLOBOCAN 2008 v1.2, Cancer Incidence and Mortality Worldwide: IARC CancerBase No. 10 [Internet] International Agency for Research on Cancer, Lyon, France, 2010. http://globocan.iarc.fr. Accessed October 7, 2012.

2. Olayioye MA, Neve RM, Lane HA and Hynes NE: The ErbB signalling network: receptor heterodimerisation in development and cancer. EMBO J 19: 3159-3167, 2000.

3. Smith I, Procter M, Dowsett M, et al; HERA study team: 2-year follow-up of trastuzumab after adjuvant chemotherapy in HER2-positive breast cancer: a randomised controlled trial. Lancet 369: 29-36, 2007.

4. Slamon DJ, Leyland-Jones B, Shak S, et al: Use of chemotherapy plus a monoclonal antibody against HER 2 for metastatic breast cancer that overexpresses HER2. N Engl J Med 344: 783-792, 2001.

5. Bernhard H1, Salazar L, Schiffman K, et al: Vaccination against the HER-2/neu oncogenic protein. Endocr Relat Cancer 9: 33-44, 2002.

6. Chen J, Li DS, Yu YH, et al: Clinical significance of Her-2 protein expression in gastric cancer. Shi Jie Hua Ren Xiao Hua Za Zhi 18: 1375-1379, 2010 (In Chinese). 
7. Ford R, Schwartz L, Dancey J, et al: Lessons learned from independent central review. Eur J Cancer 45: 268-274, 2009.

8. Takahari D, Yamada Y, Okita NT, et al: Relationships of insulin-like growth factor-1 receptor and epidermal growth factor receptor expression to clinical outcomes in patients with colorectal cancer. Oncology 76: 42-48, 2009.

9. Lim SW, Kim HR, Kim HY, et al: Over-expression of Her-2 in colorectal cancer tissue, but not in serum, constitutes an independent worse prognostic factor. Cell Oncol (Dordr) 36: 311-321, 2013.

10. Park DI, Kang MS, Oh SJ, et al: HER-2/neu overexpression is an independent prognostic factor in colorectal cancer. Int J Colorectal Dis 22: 491-497, 2007.

11. Demirbas S, Sücüllü I, Yildirim S and Celenk T: Influence of the c-erb B-2, nm23, bcl-2 and p53 protein markers on colorectal cancer. Turk J Gastroentrol 17: 13-19, 2006.

12. Kavanagh DO, Chambers G, O'Grady L, et al: Is overexpression of HER-2 a predictor of prognosis in colorectal cancer? BMC Cancer 9: 1,2009.

13. Ismail HM, El-Baradie M, Moneer M, et al: Clinico-pathological and prognostic significance of p53, Bcl-2 and Her-2/neu protein markers in colorectal cancer using tissue microarray. J Egypt Natl Canc Inst 19: 3-14, 2007.

14. Rossi HA, Liu Q, Banner B, et al: The prognostic value of invariant chain (Ii) and Her-2/neu expression in curatively resected colorectal cancer. Cancer J 8: 268-275, 2002.

15. Essapen S, Thomas H, Green M, et al: The expression and prognostic significance of HER-2 in colorectal cancer and its relationship with clinicopathological parameters. Int J Oncol 24 241-248, 2004

16. Antonacopoulou AG, Tsamandas AC, Petsas T, et al: EGFR, HER-2 and COX-2 levels in colorectal cancer. Histopathology 53: 698-706, 2008.

17. Ramanathan RK, Hwang JJ, Zamboni WC, et al: Low overexpression of HER-2/neu in advanced colorectal cancer limits the usefulness of trastuzumab (Herceptin) and irinotecan as therapy. A phase II trial. Cancer Invest 22: 858-865, 2004.

18. Kapitanović S, Radosević S, Kapitanović M, et al: The expression of p185(HER-2/neu) correlates with the stage of disease and survival in colorectal cancer. Gastroenterology 112: 1103-1013, 1997.

19. Half E, Broaddus R, Danenberg KD, et al: HER-2 receptor expression, localization, and activation in colorectal cancer cell lines and human tumors. Int J Cancer 108: 540-548, 2004.

20. Kruszewski WJ, Rzepko R, Ciesielski M, et al: Expression of HER-2 in colorectal cancer does not correlate with prognosis. Dis Markers 29: 207-212, 2010.

21. Kountourakis P, Pavlakis K Psyrri A, et al: Clinicopathologic significance of EGFR and Her-2/neu in colorectal adenocarcinomas. Cancer J 12: 229-236, 2006.

22. Schuell B, Gruenberger T, Scheithauer W, et al: HER 2/neu protein expression in colorectal cancer. BMC Cancer 6: 123, 2006.

23. Kim JY, Lim SJ and Park K: Cyclooxygenase-2 and c-erbB-2 expression in colorectal carcinoma assessed using tissue microarray. Appl Immunohistochem Mol Morphol 12: 67-70, 2004.

24. Marx AH, Burandt EC, Choschzick M, et al: Heterogenous high-level HER-2 amplification in a small subset of colorectal cancers. Hum Pathol 41: 1577-1585, 2010.

25. Herreros-Villanueva M, Rodrigo M, Claver M, et al: KRAS, BRAF, EGFR and HER2 gene status in a Spanish population of colorectal cancer. Mol Biol Rep 38: 1315-1320, 2011

26. Pappas A, Lagoudianakis E, Seretis C, et al: Clinical role of HER-2/neu expression in colorectal cancer. J BUON 18: 98-104, 2013.

27. Li Q, Wang D, Li J and Chen P: Clinicopathological and prognostic significance of HER-2/neu and VEGF expression in colon carcinomas. BMC Cancer 11: 277, 2011

28. Seo AN, Kwak Y,Kim DW, et al: HER2 Status in colorectal cancer: its clinical significance and the relationship between HER2 gene amplification and expression. PLoS One 9: e98528, 2014.

29. Sobin LH and Fleming ID: TNM classification of malignant tumors, fifth edition (1997). Union Internationale Contre le Cancer and the American Joint Committee on Cancer. Cancer 80: 1803-1804, 1997.
30. Hamtilon SR and Aaltonen LA (eds): World Health Organization classification of tumours. Tumours of the oesophagus. In: Pathology and genetics tumors of the digestive system. IARC Press, Lyon, 2006

31. Hofmann M, Stoss O, Shi D, et al: Assessment of a HER2 scoring system for gastric cancer: results from a validation study. Histopathology 52: 797-805, 2008.

32. Pauletti G, Dandekar S, Rong H, et al: Assessment of methods for tissue-based detection of the HER-2/neu alteration in human breast cancer: a direct comparison of fluorescence in situ hybridization and immunohistochemistry. J Clin Oncol 18: 3651-3664, 2000.

33. Tavangar SM, Shariftabrizi A and Soroush AR: Her-2/neu over-expression correlates with more advanced disease in Iranian colorectal cancer patients. Med Sci Monit 11: CR123-CR126, 2005.

34. McKay JA, Loane JF, Ross VG, et al: c-erbB-2 is not a major factor in the development of colorectal cancer. Br J Cancer 86 : $568-573,2002$

35. Mann M, Sheng H, Shao J, et al: Targeting cyclooxygenase 2 and HER-2/neu pathways inhibits colorectal carcinoma growth. Gastroenterology 120: 1713-1719, 2001.

36. Pohl M, Stricker I, Schoeneck A, et al: Antitumor activity of the HER2 dimerization inhibitor pertuzumab on human colon cancer cells in vitro and in vivo. J Cancer Res Clin Oncol 135: 1377-1386, 2009.

37. Liu W, Zhong S, Chen J and Yu Y: HER-2/neu overexpression is an independent prognostic factor for intestinal-type and early-stage gastric cancer patients. J Clin Gastroenterol 46: e31-e37, 2012.

38. Rossi E, Ubiali A, Cadei M, et al: HER-2/neu in breast cancer: a comparative study between histology, immunohistochemistry, and molecular technique (FISH). Appl Immunohistochem Mol Morphol 14: 127-131, 2006.

39. Chibon F, de Mascarel I, Sierankowski G, et al: Prediction of HER2 gene status in HER2 2+ invasive breast cancer: a study of 108 cases comparing ASCO/CAP and FDA recommendations. Mod Pathol 22: 403-409, 2009.

40. Bartlett JM, Going JJ, Mallon EA, et al: Evaluating HER2 amplification and overexpression in breast cancer. J Pathol 195: 422-428, 2001

41. Downs-Kelly E, Yoder BJ, Stoler M, et al: The influence of polysomy 17 on HER2 gene and protein expression in adenocarcinoma of the breast: a fluorescent in situ hybridization, immunohistochemical, and isotopic mRNA in situ hybridization study. Am J Surg Pathol 29: 1221-1227, 2005.

42. Dal Lago L, Durbecq V, Desmedt C, et al: Correction for chromosome-17 is critical for the determination of true Her-2/neu gene amplification status in breast cancer. Mol Cancer Ther 5: 2572-2579, 2006

43. Winston JS, Ramanaryanan J and Levine E: HER-2/neu evaluation in breast cancer are we there yet? Am J Clin Pathol 121 (Suppl): S33-S49, 2004.

44. Seidal T, Balaton AJ and Battifora H: Interpretation and quantification of immunostains. Am J Surg Pathol 25: 1204-1207, 2001.

45. Amado RG, Wolf M, Peeters M, et al. Wild-type KRAS is required for panitumumab efficacy in patients with metastatic colorectal cancer. J Clin Oncol 26: 1626-1634, 2008.

46. Di Nicolantonio F, Martini M, Molinari F, Sartore-Bianchi A, et al. Wild-type BRAF is required for response to panitumumab or cetuximab in metastatic colorectal cancer. J Clin Oncol 26: 5705-5712, 2008.

47. Liu W, Wang L, Yu YH, et al: Detection of k-ras gene mutations in Chinese patients with colorectal cancer. World Chinese Journal of Digestology 19: 1367-1374, 2011

48. LaBonte MJ,Wilson PM,Fazzone W, et al: The dualEGFR/HER2 inhibitor lapatinib synergistically enhances the antitumor activity of the histone deacetylase inhibitor panobinostat in colorectal cancer models. Cancer Res 71: 3635-3648, 2011.

49. Chen J, Li Q, Wang C, Wu J and Zhao G: Prognostic significance of c-erbB-2 and vascular endothelial growth factor in colorectal liver metastases. Ann Surg Oncol 17: 1555-1563, 2010. 